\title{
A Novice-Friendly Induction Tactic for Lean
}

\author{
Jannis Limperg \\ Vrije Universiteit Amsterdam \\ Department of Computer Science \\ Amsterdam, The Netherlands \\ j.b.limperg@vu.nl
}

\begin{abstract}
In theorem provers based on dependent type theory such as Coq and Lean, induction is a fundamental proof method and induction tactics are omnipresent in proof scripts. Yet the ergonomics of existing induction tactics are not ideal: they do not reliably support inductive predicates and relations; they sometimes generate overly specific or unnecessarily complex induction hypotheses; and they occasionally choose confusing names for the hypotheses they introduce.

This paper describes a new induction tactic, implemented in Lean 3, which addresses these issues. The tactic is particularly suitable for educational use, but experts should also find it more convenient than existing induction tactics. In addition, the tactic serves as a moderately complex case study for the metaprogramming framework of Lean 3 . The paper describes some difficulties encountered during the implementation and suggests improvements to the framework.
\end{abstract}

CCS Concepts: • Mathematics of computing $\rightarrow$ Mathematical software; • Theory of computation $\rightarrow$ Type theory; Logic and verification.

Keywords: induction, tactic, Lean, metaprogramming, type theory

\section{ACM Reference Format:}

Jannis Limperg. 2021. A Novice-Friendly Induction Tactic for Lean. In Proceedings of the 10th ACM SIGPLAN International Conference on Certified Programs and Proofs (CPP '21), fanuary 18-19, 2021, Virtual, Denmark. ACM, New York, NY, USA, 13 pages. https://doi. org/10.1145/3437992.3439928

\section{Introduction}

Induction is a fundamental proof method in dependently typed interactive theorem proving, and so in proof assistants

Permission to make digital or hard copies of all or part of this work for personal or classroom use is granted without fee provided that copies are not made or distributed for profit or commercial advantage and that copies bear this notice and the full citation on the first page. Copyrights for components of this work owned by others than the author(s) must be honored. Abstracting with credit is permitted. To copy otherwise, or republish, to post on servers or to redistribute to lists, requires prior specific permission and/or a fee. Request permissions from permissions@acm.org. CPP '21, January 18-19, 2021, Virtual, Denmark

( 2021 Copyright held by the owner/author(s). Publication rights licensed to ACM.

ACM ISBN 978-1-4503-8299-1/21/01 ..\$15.00

https://doi.org/10.1145/3437992.3439928 such as Coq and Lean, the induction tactic is usually among the first tactics a novice encounters. Yet despite this elevated position, current induction tactics exhibit a number of usability issue. Experts have got used to these dark corners and routinely perform the busywork required to get around them. Newcomers to dependently typed theorem proving, however, often struggle to connect the workings of the induction tactic with their informal understanding of proof by induction. Jasmin Blanchette and colleagues noticed this when designing the Logical Verification course at Vrije Universiteit Amsterdam, which uses Lean to teach interactive theorem proving. They had to devote significant space and time to technical issues with Lean's standard induction tactic, which distracted from more fundamental topics.

To shield novices (and experts) from these distractions, we need induction tactics which minimise the gap between formal and informal proof by induction. This paper describes an attempt at such a tactic. In particular, it addresses three usability issues with existing induction tactics.

Indexed inductive types. The standard induction tactics of Coq and Lean sometimes produce counterintuitive goals when used with indexed inductive types. Consider the type fin $n$ of natural numbers strictly less than $n$, which can be encoded as an indexed inductive type. Given this encoding, it is trivial on paper to prove by induction (or even mere case distinction) that fin $\theta$ is uninhabited. But when we apply the standard induction tactics of Coq and Lean to this goal, they produce a goal which asks us, unhelpfully, to prove false in an empty context. Similar problems occur regularly when formalising programming language metatheory, which tends to use indexed inductive types extensively to define inductive predicates and relations.

Coq and Lean both provide case splitting tactics which prove that $f$ in $\theta$ is uninhabited, but which cannot deal with goals that require induction. While this is obviously useful, it is pedagogically unfortunate: case splitting should produce the same goals as induction, only without the induction hypotheses. This connection is obscured if case splitting and induction tactics handle indexed types differently.

Coq (but not Lean) also provides dependent induction, an alternative induction tactic which handles indexed inductive types better. It proves our lemma about $\mathrm{f}$ in 0 immediately. But this tactic creates a new issue: it often produces unnecessarily complex induction hypotheses. In one of the 
examples I discuss below, dependent induction creates the induction hypothesis

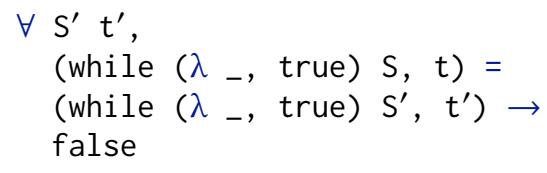

which is obviously equivalent to false.

My induction tactic works like dependent induction, but simplifies the induction hypotheses to eliminate redundant arguments. This combination yields intuitive goals in many situations involving indexed induction types.

Overly specific induction hypotheses. Standard induction tactics tend to produce overly specific induction hypotheses. Consider this humble injectivity lemma:

$\forall(\mathrm{n} m: \mathbb{N}), \mathrm{n}+\mathrm{n}=\mathrm{m}+\mathrm{m} \rightarrow \mathrm{n}=\mathrm{m}$

If we perform induction on $n$ after introducing $n, m$ and the equation, we get an induction hypothesis about a fixed $\mathrm{m}$ :

$\mathrm{n}+\mathrm{n}=\mathrm{m}+\mathrm{m} \rightarrow \mathrm{n}=\mathrm{m}$

Unfortunately, this induction hypothesis does not help us make progress with the proof. Instead, we need an induction hypothesis which generalises over all $\mathrm{m}$ :

$\forall \mathrm{m}, \mathrm{n}+\mathrm{n}=\mathrm{m}+\mathrm{m} \rightarrow \mathrm{n}=\mathrm{m}$

Convincing Coq or Lean to generate the more general induction hypothesis is not hard. We can introduce $m$ after the induction (rather than before) or use special syntax offered by the standard induction tactics. However, this still presents a problem for novices: they must first recognise that the original induction hypothesis is not helpful and that generalising $m$ is the appropriate remedy. Neither of these insights will be obvious to someone not yet familiar with the mechanics of interactive theorem proving.

That is why my induction tactic does the opposite of the standard tactics: rather than generating the most specific induction hypotheses by default, it generates the most general ones. In effect, it generalises every hypothesis like we generalised $\mathrm{m}$, with some restrictions to avoid obviously useless generalisations. This sometimes produces overly general induction hypotheses, but for novices, that is a better problem to have. They can simply specialise an induction hypothesis to recover the more specific version.

Naming. Induction tactics generate many new hypotheses and these hypotheses must be named. This may seem like a trivial concern, but Lean's standard induction tactic shows that it is not. Consider this simple lemma about a particular formulation of the transitive closure tc $r$ of a binary relation $r$ (which in Lean would usually live in the universe Prop of propositions, but for simplicity I pretend throughout that there is only one universe Type):

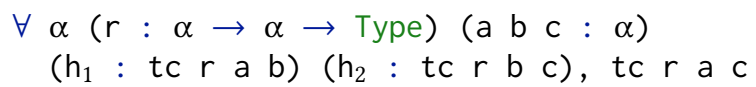

When we use Lean's standard induction tactic to perform induction on $h_{1}$, it generates a rather forbidding goal:

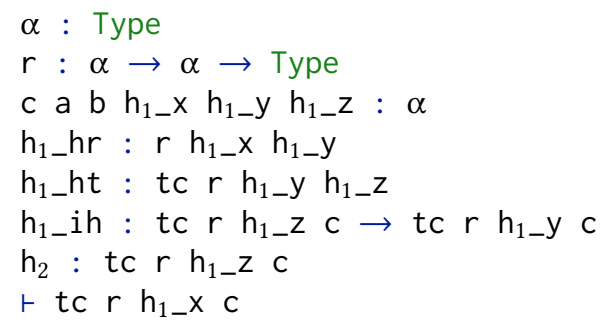

The new hypotheses' names are clearly too cumbersome: experts and novices alike will want to immediately rename almost everything. Worse, the names are misleading because the first element of the transitive chain, $a$, is now $h_{1 \_} x$ while b has become $h_{1 \_z}$. Novices will struggle to make the connection between the old and new hypotheses, and thus to understand how this goal is connected to the lemma they wanted to prove. Coq's standard induction tactic generates more helpful names than Lean's, but it too misses this connection.

To prevent the resulting confusion, my induction tactic uses a number of heuristics to generate names that reflect common intuitions about how induction works. For the tc example, it generates this goal:

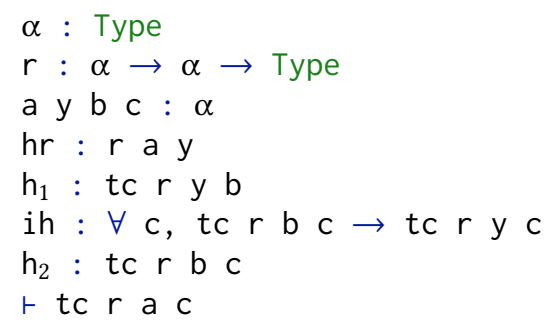

Omitting the $h_{1}$ prefixes and preserving the names $a, b$ and c makes for a much more reasonable-looking goal.

The new induction tactic, which addresses the above usability issues, is described in Sect. 3, after I lay some terminological groundwork in Sect. 2. The description proceeds chronologically through every step the new induction tactic takes when processing a goal.

The new induction tactic could be implemented in any dependently typed proof assistant with indexed inductive types and a suitable metaprogramming framework. (Parts of it do, however, make use of the somewhat controversial axiom K.) An implementation for Lean 3 is available in mathlib [5], Lean's de facto standard library. ${ }^{1}$ The tactic was also used in the 2020 edition of the Logical Verification course, where it replaced Lean's standard induction tactic. This allowed the course authors to significantly simplify the lecture

\footnotetext{
${ }^{1}$ The version of mathlib which contains the implementation described in this paper is available online at https://github.com/leanprover-community/ mathlib/tree/d36af184d154f2e99f60fec5cd71bb3e53899d5c. The relevant source file is src/tactic/induction. lean. An archive of this code is available at https://doi.org/10.5281/zenodo.4327209.
} 
notes and accompanying code, since the tricks experts use to make Lean's standard induction tactic work did not need to be taught any more.

The new induction tactic is among the larger tactics written in Lean's metaprogramming framework [7]. This provides an opportunity to evaluate how the framework fares on a moderately complex task. In Sect. 4, I describe some problems I encountered while implementing the tactic, as well as possible workarounds and suggestions for improvements. This case study will hopefully be useful to aspiring Lean metaprogrammers and may guide the design of the metaprogramming framework in the upcoming fourth version of Lean.

In summary, I make the following contributions:

- I describe an induction tactic that is more ergonomic than the state of the art. The design is geared particularly towards novice users, but experts should also find it easier to work with.

- I provide an implementation of this tactic in Lean 3, which previously lacked a convenient induction tactic.

- I give an experience report about Lean's metaprogramming framework, pointing out some pitfalls and suggesting improvements.

\section{Induction in Dependent Type Theory}

Induction in dependent type theories is intimately connected with indexed inductive types [6], a fundamental concept of most modern dependently typed theorem provers. Indexed inductive types generalise non-indexed inductive types such as natural numbers and lists and are often used to define inductive predicates and relations.

A typical example of this use is the transitive closure of a binary relation, which can be encoded in Lean as follows:

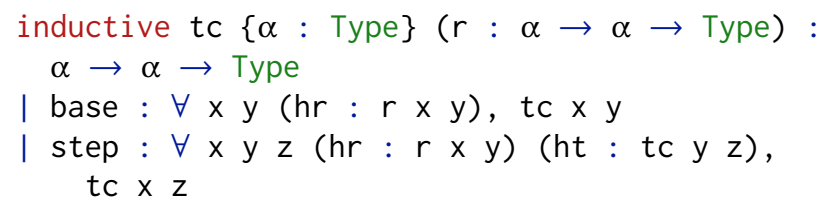

The above defines a type family tc of type

$\forall\{\alpha:$ Type $\}(r: \alpha \rightarrow \alpha \rightarrow$ Type $)$, $\alpha \rightarrow \alpha \rightarrow$ Type

The first argument of tc, $\alpha$, will be left implicit, as indicated by the curly braces. The second argument is the relation $r$ whose transitive closure we are taking. The third and fourth arguments are elements of $\alpha$ that are related by tc $r$.

The transitive closure is inductively generated by two rules corresponding to the two constructors of tc. The base constructor says that if two elements $x$ and $y$ are related by $r$, then they are also related by tc $r$. The step constructor says that if $r$ relates $x$ and $y$, and tc $r$ relates $y$ and $z$, then tc $r$ relates $x$ and $z$.

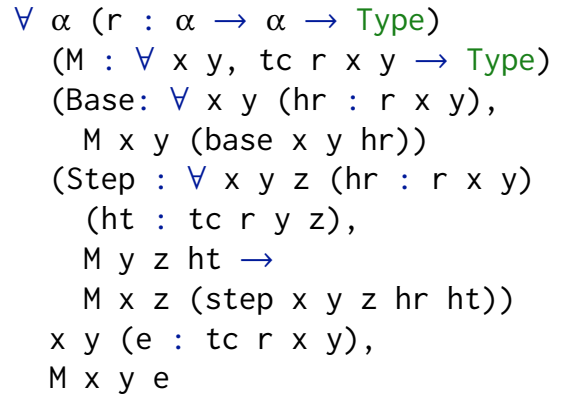

Figure 1. The type of tc. rec

In the type of tc, we distinguish between two kinds of arguments: parameters and indices. Arguments that appear before the colon, here $\alpha$ and $r$, are parameters of tc. Parameters are implicitly quantified over in the types of tc's constructors, and whenever tc appears in a constructor type, it is implicitly applied to the parameters. Thus, the full type of base is

$\forall\{\alpha\}\{r: \alpha \rightarrow \alpha \rightarrow$ Type $\}(x \mathrm{y}: \alpha)$,
$\quad r \mathrm{x}$ y $\rightarrow$ tc $r \mathrm{x}$ y

The arguments of tc after the colon are its indices. Unlike parameters, these may vary freely in the constructor types, and indeed our constructors instantiate the indices of tc with different expressions.

Each inductive type has an associated induction principle, the (dependent) recursor, which reflects the fact that every closed element of the inductive type consists of finitely many constructor applications. In Lean, a recursor is added as an axiom whenever we define an inductive type [1]. For tc, we get the recursor tc. rec, whose type appears in Fig. 1. This type is derived from tc as follows:

- The first two arguments, $\alpha$ and $r$, are the parameters of tc.

- $M$ is the type we are constructing, also known as the motive of the induction. It is a predicate over elements of tc (and its indices).

- Base and Step are minor premises corresponding to the constructors of $t c$. They ask users to give one proof of $M$ for the case where tc $r \times y$ was proved by base and one for the case where tc $r x y$ was proved by step. In the step case, we may assume a proof of $M$ for the recursive constructor argument ht.

- From all this data, tc. rec concludes $M x$ y e for an arbitrary element e of tc $r \times \mathrm{y}$. We call e the major premise of the induction. This is the hypothesis on which we perform induction.

To perform induction in Lean, then, means to apply the recursor of an inductive type. For an example, we return to the transitivity of the transitive closure: 


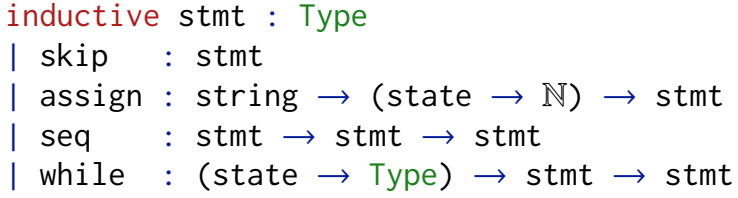

Figure 2. Syntax of a toy imperative language

$\forall \alpha(r: \alpha \rightarrow \alpha \rightarrow$ Type $)(x$ y z : $\alpha)$

(hxy : tc $r \times y$ ) (hyz : tc $r$ y z), tc $r \times z$

We first fix $\alpha, r, x, y$ and $z$. The proof then proceeds by recursion on hxy, so this is our major premise. The parameters, $\alpha$ and $r$, are already determined by this choice, as are the major premise indices $x$ and $y$. For the motive, we choose

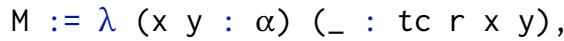

tc $r y z \rightarrow$ tc $r \times z$

Substituting this motive in the minor premises, we are left with one proof obligation for each constructor, corresponding to the cases of the induction. The proof of our lemma then reads:

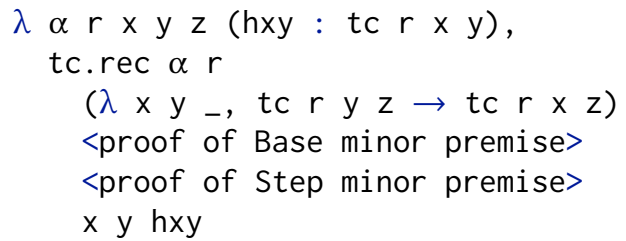

An induction tactic helps with this rather arduous exercise by automating much of it. Ideally, users do not have to contend with motives, parameters or indices and are presented only with one intuitive new goal for each constructor. The next section explains how to achieve this in many cases.

\section{Implementation of the Induction Tactic}

The following subsections describe each step the new induction tactic takes to perform an induction, in chronological order.

\subsection{Generalisation of Complex Indices}

The first problem our induction tactic must solve is the treatment of complex index arguments in a major premise. To see what this means, consider a typical task in programming language metatheory: a simple lemma about the big-step semantics of a toy imperative language.

The abstract syntax of our toy language is defined by the (non-indexed) inductive type stmt in Fig. 2. Its constructors represent, from top to bottom, a no-op statement; variable assignment; sequencing of statements; and a while loop. The state type mentioned by some constructors represents the current program heap as a map from variable names to their current values (which, for simplicity, are always natural numbers). The loop condition of a while loop is given as a predicate on the heap state.

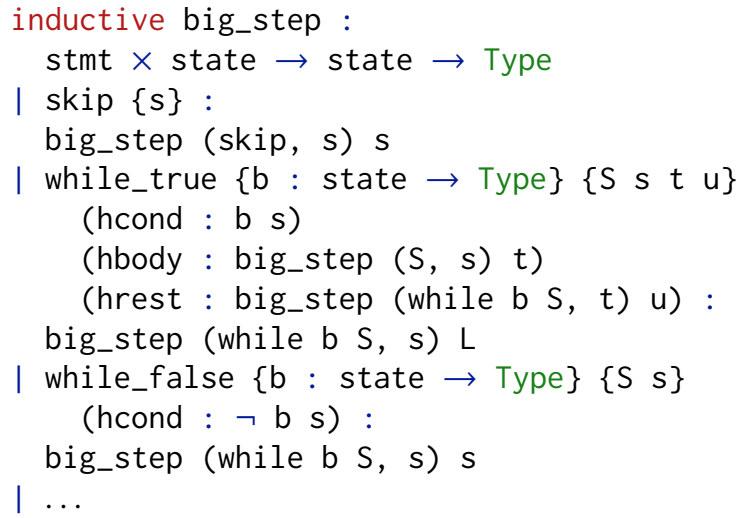

Figure 3. Big-step semantics of the toy language

The language's big-step semantics are given by the indexed inductive type big_step in Fig. 3, omitting some constructors. This type defines a relation between a program $S$, an initial state $s$ and a final state $t$. If big_step $(S, s) t$ is derivable, then $S$, when executed in heap state $s$, terminates in heap state $t$. We write $(S, s) \Rightarrow t$ for big_step $(S, s) t$. To enable this notation in Lean 3, and following standard informal practice, the first argument of big_step is a pair type (so big_step is partially uncurried).

Now we want to prove that the infinite loop does not terminate. In Lean, this means solving the following goal:

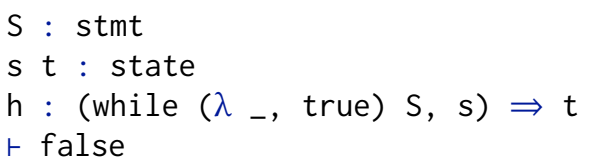

Above the turnstile appear the local hypotheses of our goal, most importantly $h$, which says that the infinite loop steps to some state $t$ and thus terminates. Right of the turnstile is our target, the canonical empty type false. On paper, this goal is easily proven by induction on the derivation of $h$. Lean and Coq's default induction tactics, however, fail us. Applying them yields unprovable subgoals.

This is because in the type of $\mathrm{h}$, big_step has a complex index. A term is complex if it is anything other than a local hypothesis. If such a term appears as an index of an inductive type, trouble ensues. Here, the offending complex index is the first argument of big_step,

(while $\left(\lambda_{-}\right.$, true) $\left.s, s\right)$

A naive induction tactic now proceeds as follows. Our target is false, which depends neither on the hypothesis $h$ nor its indices, so the motive of the induction is the constant function

$$
\begin{gathered}
M:=\lambda(x: \text { stmt } \times \text { state })(t: \text { state }) \\
(p: x \Rightarrow t), \text { false }
\end{gathered}
$$


Constructing the type of big_step's recursor according to the schema from Sect. 2, we get the following minor premise for the skip constructor:

$\forall$ (s: state), M (skip, s) s skip

Yet this gives a plainly unprovable goal if we substitute the motive M:

$\forall$ (s: state), false

The root cause of this issue is that by applying the recursor like we did, we effectively forgot that the first index of the major premise involved a while, not a skip. As a result, we cannot recognise that the major premise could not have been constructed by big_step's skip constructor.

This deficiency of induction tactics in the presence of complex indices is well known. The traditional solution, in the context of dependent type theory, is due to McBride [11]. The remainder of this section describes a variant of his procedure, with one major change. McBride's tactic analyses arbitrary elimination principles, determines which of their arguments lead to problems similar to our complex index problems and generalises those arguments. In contrast, we only support the standard recursors of inductive types, for which we know that issues like the one we have seen are only caused by complex indices. This makes our tactic less general, but it also considerably simplifies the implementation (and its presentation below). In an educational setting, where custom elimination principles are rarely used, this seems like an acceptable trade-off. Coq's dependent induction uses a very similar restricted variant of McBride's approach (which is, to my knowledge, not described in the literature). Coq does support custom elimination principles with dependent induction, but in this case the tactic still only generalises complex indices. This capability could also be added, with moderate engineering effort, to our tactic.

McBride's solution to the complex index problem is to replace any complex index $\mathrm{i}$ with a new hypothesis $\mathrm{Hi}$, called an index placeholder, and to add an index equation $\mathrm{Hi}=\mathrm{i}$ to the target. This ensures that we do not lose information about the value of the index. Applying this transformation yields an equivalent goal:

$S$ : stmt

$s \mathrm{t}$ : state

$\mathrm{Hi}:$ stmt $\times$ state

$\mathrm{h}: \mathrm{Hi} \Rightarrow \mathrm{t}$

$\vdash \mathrm{Hi}=\left(\right.$ while $\left(\lambda_{-}\right.$, true) $\left.\mathrm{s}, \mathrm{s}\right) \rightarrow$ false

Then we proceed as before. But since our goal now depends on the first index of $h$, we generate a different motive for the induction:

$\lambda$ ( $x$ : stmt $\times$ state) (t : state) ${ }_{-}$, $x=\left(\right.$ while $\left(\lambda_{-}\right.$, true $\left.) \mathrm{s}, \mathrm{s}\right) \rightarrow$ false

The minor premise for skip changes accordingly, leaving us with this goal for the skip case:
$S:$ stmt

$\mathrm{s} \mathrm{s}^{\prime}$ : state

ieq $:\left(\right.$ skip, $\left.s^{\prime}\right)=\left(\right.$ while $\left(\lambda_{-}\right.$, true $\left.) s, s\right)$

$\vdash$ false

This goal is provable because the equation ieq, which is derived from the index equation, is contradictory. We have, in effect, remembered that the index of big_step was a while, not a skip.

To make this index generalisation procedure work for more complex goals, we must address two technical complications. First, when we replace a complex index in the major premise, we generally want to also replace it in the target and in the types of other hypotheses, to make sure that the goal remains type-correct. This is a somewhat crude heuristic since the replacement may itself introduce type errors, but it is right more often than wrong. However, we never replace the index in hypotheses that occur in the type of the major premise.

A second complication arises when there are dependencies between the indices of an inductive family. Consider, for example, the family

$F: \forall(x: X)(y: Y x)$, Type

where $x$ and $y$ are indices, and suppose that we want to perform induction on the hypothesis $h: F t u$ (with $t$ and $u$ complex terms). The index generalisation procedure then replaces $\mathrm{t}$ with a new hypothesis $\mathrm{Ht}: \mathrm{X}$ such that $\mathrm{Ht}=\mathrm{t}$ and $\mathrm{u}$ with a new hypothesis $\mathrm{Hu}: \mathrm{YHt}$ such that $\mathrm{Hu}=\mathrm{u}$. But this last equation is not well-typed: $u$ has type $Y t$, not $Y \mathrm{Ht}$. In this situation, we must use a heterogeneous equation, written $\mathrm{Hu}==\mathrm{u}$, where the two sides may have different types.

At this point, one might become concerned for novice users of the induction tactic: would they not get overwhelmed with index placeholders and index equations? Fortunately, Sect. 3.3 shows that the new hypotheses can usually be eliminated automatically after we have applied the recursor, so our users do not get to see them.

\subsection{Generalisation of Induction Hypotheses}

One of the more arcane aspects of Coq and Lean's existing induction tactics is that they ask their users to specify which hypotheses can vary during the induction and which are fixed. This leads to counterintuitive behaviour even in simple cases. Pierce [13] illustrates the problem with the following injectivity lemma:

$\forall(\mathrm{n} m: \mathbb{N}), \mathrm{n}+\mathrm{n}=\mathrm{m}+\mathrm{m} \rightarrow \mathrm{n}=\mathrm{m}$

An unsuspecting novice will mechanically introduce $n, m$ and the equation, then perform induction on $n$. This produces the following goal for the successor case:

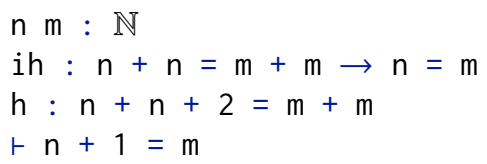


Unfortunately, this gives us an induction hypothesis, ih, that is not applicable: it presumes $n=m$ when we have, according to $h, n=m-1$. The solution is to let $m$ vary during the induction instead of keeping it fixed, which gives a more sensible goal:

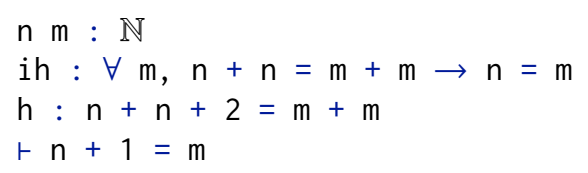

Now ih can be instantiated with $m-1$ to close the goal.

Generalising the induction hypothesis in this manner is not difficult, and existing tactics provide special syntax for it. But to a novice, it will be far from obvious that this is why our first proof attempt gets stuck. Novices often have trouble recognising that a goal is unprovable in the first place, and when they do, they may suspect any number of errors on their part. A novice-friendly induction tactic should therefore not fix every hypothesis by default, as the existing tactics do, but rather generalise every hypothesis. This sometimes leads to an overly general induction hypothesis, but that is much less harmful: the user does not get stuck but merely has to apply the induction hypothesis to some additional arguments. Our new tactic also offers a convenient syntax to fix some or all hypotheses; if all hypotheses are fixed, the tactic behaves like the existing induction tactics.

Implementing this automatic generalisation is straightforward in most cases. We simply revert ("unintroduce") all hypotheses before applying the recursor. However, there are three classes of hypotheses that should not be reverted:

1. Hypotheses which the user has explicitly fixed and their dependencies, i.e. those hypotheses which occur in the type of a fixed hypothesis. If we were to revert a dependency of a fixed hypothesis, we would also have to revert the fixed hypothesis.

2. Hypotheses on which the major premise depends. Such hypotheses cannot be reverted without also reverting the major premise.

3. Hypotheses which would not make the induction hypotheses more general if we were to revert them.

The last class deserves further analysis. Usually, when we perform an induction, all hypotheses are relevant to the proof, so generalising a hypothesis leads to a more general induction hypothesis. However, that is not always the case. In longer proofs, it is occasionally convenient to prove a helper lemma (by induction) inline, without leaving the proof environment. These lemmas may involve only some of the hypotheses, but if we follow our generalise-everything approach, we also generalise all the other hypotheses in the context which have nothing to do with the helper lemma. This gives us an induction hypothesis with additional redundant arguments.

Consider this example: $x: X$

$\mathrm{n} \mathrm{m}: \mathbb{N}$

$r \mathrm{n}+\mathrm{m}=\mathrm{m}+\mathrm{n}$

The first hypothesis, $x$, has nothing to do with the rest of the goal - perhaps we were in the middle of a proof involving $x$ and decided to prove commutativity of addition inline as a helper lemma. To that end, we perform induction on $n$. The naive generalisation algorithm would now revert both $x$ and $\mathrm{m}$, yielding an induction hypothesis with an obviously redundant argument:

$\forall(x: X)(m: \mathbb{N}), n+m=m+n$

To prevent this, we revert a hypothesis $\mathrm{h}$ only if it meets at least one of the following criteria:

1. $\mathrm{h}$ occurs in the target. Recall that the motive of the induction, which determines the induction hypothesis, is derived from the target. So if the target has the form $\forall \mathrm{x}, \mathrm{P} \mathrm{x}$ instead of $\mathrm{P} \mathrm{h}$, we get a more general induction hypothesis. In the commutativity example, $m$ fulfils this criterion, so it is generalised.

2. $h$ depends on the major premise, or on any of the dependencies of the major premise. Then, $h$ is likely to be a property of the major premise that is relevant to the induction. For instance, if we have a major premise $\mathrm{n}: \mathbb{N}$, a hypothesis $\mathrm{h}: \mathrm{n}>0$ and a target $\mathrm{P} n$, the motive of the induction should be derived from the generalised target $n>0 \rightarrow P n$. Otherwise we would not be able to use the fact that $n>0$ during the induction, and in particular we would not be able to discharge the case for $n=0$ by noting that $\theta \ngtr \theta$. The same reasoning also applies to dependencies of the major premise: performing the induction may give us additional information about these dependencies, so hypotheses mentioning them may be of interest.

Conversely, any hypothesis $\mathrm{h}$ that does not meet either criterion - such as $\mathrm{x}$ in the commutativity example - should not be generalised. Such hypotheses have no connection to either the target or the major premise, so generalising them only adds redundant arguments to the induction hypothesis. Of course, our criteria only prevent the most obvious forms of over-generalisation: for the commutativity proof, $m$ does not need to be generalised either.

This step concludes the preprocessing, so now our tactic applies the recursor. To do so, we would usually have to generate a motive, which involves solving a higher-order unification problem. Luckily, Lean has a built-in heuristic that generates correct motives most of the time, so we do not have to concern us with this issue here.

By applying the recursor, we generate one new goal for each case of the induction (i.e. each minor premise). The next steps are applied to each of these goals individually. 


\subsection{Unification of Index Equations}

In Sect. 3.1, we introduced placeholders for the complex indices of the major premise and index equations to remember what the placeholders stand for. We can usually eliminate these equations again after the recursor has been applied, using McBride's Qnify tactic [10].

Qnify implements a form of first-order unification. It works on a queue of equations which initially contains the equations for each index, starting with the first. The order is important when indices depend on each other since unification of earlier index equations may simplify later ones. The two sides of each equation are unified by applying the following set of rules until no rule applies any more:

Substitution. For an equation eq : $x=t$ or eq : $t=x$, where $x$ is a local hypothesis and $t$ is a term in which $x$ does not occur, delete eq and replace $x$ with $t$ everywhere in the goal.

Injection. For eq : $C t_{1} \ldots t_{n}=C u_{1} \ldots u_{n}$, where $C$ is a constructor of an inductive type, delete eq and add new equations $t_{i}=u_{i}$. The new equations are added to the front of the queue, so they are processed immediately after this step. Some of the equations may have to be heterogeneous.

Conflict. For eq : $C t_{1} \ldots t_{n}=D u_{1} \ldots u_{m}$, where $C$ and $\mathrm{D}$ are distinct constructors, solve the goal since eq is contradictory.

Deletion. For eq : $t=u$, where $t$ and $u$ are definitionally equal, delete eq.

Cycle. For eq : $\mathrm{x}=\mathrm{t}$ (or symmetric), where $\mathrm{x}$ appears under constructors in $t$, solve the goal since eq is contradictory. The previous condition means that $t$ must be of the form

$\mathrm{C}_{1} \ldots\left(\mathrm{C}_{2} \ldots\left(\mathrm{C}_{n} \ldots \times \ldots\right) \ldots\right) \ldots$

where the $C_{i}$ are all constructors of the same inductive type and $n$ is not zero. For example, this rule would match the equation $x=\operatorname{succ}(\operatorname{succ}(\operatorname{succ} x))$, where succ is the successor constructor of $\mathbb{N}$.

Homogenisation. For eq : $t==u$, where $t: T, u: U$ and $T$ is definitionally equal to $U$, replace eq with the equivalent homogeneous equation $t=u$. This rule typically applies because the types $T$ and $U$ were initially distinct - hence the heterogeneous equation $t==u-$ but became definitionally equal during the unification of earlier equations.

Recall the example from Sect. 3.1. After generalising complex indices, we ended up with this goal in one of the cases of the induction:

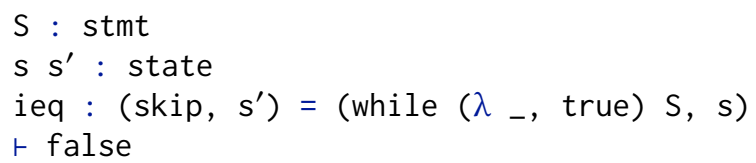

Applying Qnify to the index equation ieq, we first use the injection rule since both sides of the equation are applications of the pair constructor $\left({ }_{-},{ }_{-}\right)$. This gives us new equations:

ieq $_{1}:$ skip $=$ while $\left(\lambda_{-}\right.$, true $) \mathrm{S}$

ieq $_{2}: s^{\prime}=s$

We then apply the conflict rule to ieq $_{1}$ since skip and while are different constructors, solving the goal. Thus, users of our tactic never get to see this case of the induction.

The homogenisation rule, which deals with heterogeneous equations, is only valid in certain type theories, namely those in which Streicher's axiom K [8] is derivable. This includes Lean, but excludes some other popular proof assistants, particularly those that seek to be compatible with the univalence axiom of homotopy type theory [14], which is incompatible with axiom $\mathrm{K}$. Induction tactics for such type theories would not use McBride's index generalisation method but rather that of Cockx et al. [3, 4], who show how to achieve a similar effect without using axiom $\mathrm{K}$.

Implementing the unification procedure is straightforward except for the cycle rule. To prove that an equation

eq : $\mathrm{x}=\mathrm{C}_{1}\left(\ldots\left(\mathrm{C}_{n} \mathrm{x}\right) \ldots\right)$

is contradictory, we use a size measure sizeof which counts the number of constructors in a term. Lean generates this measure for every inductive type. Applying it on both sides of the equation yields an equation in $\mathbb{N}$ :

eq : sizeof $x=$ sizeof $x+n$

For positive $\mathrm{n}$, this can be discharged by applying an appropriate lemma.

\subsection{Simplification of Induction Hypotheses}

The index placeholders and index equations introduced in Sect. 3.1 also occur as additional arguments to the induction hypotheses generated by the recursor application. But like the equations themselves, these arguments can often be trivially eliminated. Prior work does not address this issue: Coq's dependent induction tactic makes no attempt to simplify the induction hypotheses and Lean's cases tactic, which also uses McBride's index generalisation technique, does not generate induction hypotheses in the first place.

Let us again consider the big_step example from Sect. 3.1, but now we focus on the first inductive case. After the index equations have been eliminated, we get the goal shown in Fig. 4, corresponding to the while_true constructor of big_step.

The induction hypotheses, $i h_{1}$ and $i h_{2}$, have been generalised over two index placeholders, $S^{\prime}$ and $t^{\prime}$, and an index equation. But in $i h_{2}$, these are all redundant. We can only hope to apply $i h_{2}$ if we instantiate $S^{\prime}$ with $S$ and $t^{\prime}$ with $t$; any other instantiation (modulo propositional equality) would not satisfy the index equation.

This is a common case, so we postprocess the induction hypotheses to eliminate such redundant arguments. To do 


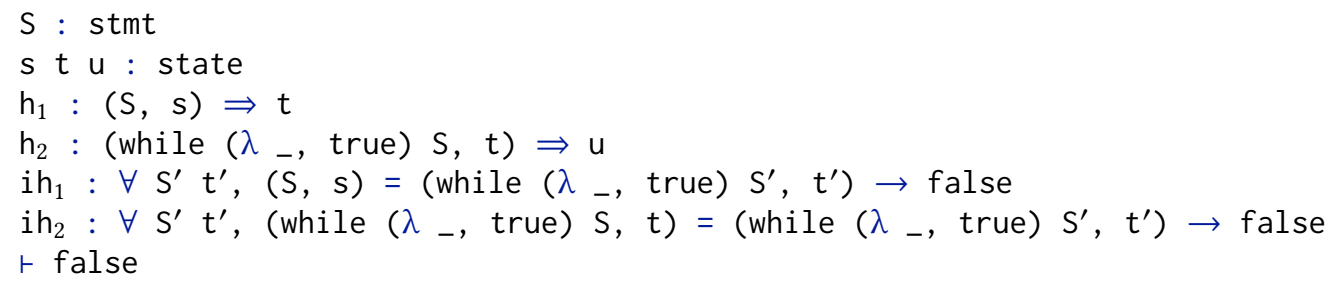

Figure 4. A goal before simplification of induction hypotheses

so, we first replace each index placeholder in the type of $\mathrm{ih}_{2}$ with a fresh metavariable:

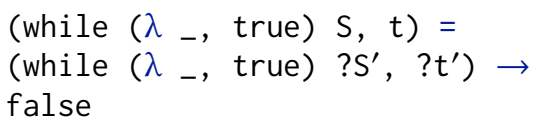

We then iterate through the index equations - here only one - and unify the left-hand side of each with the righthand side, using Lean's built-in unification procedure. If unification finds a unique solution, the metavariables are assigned accordingly:

$? \mathrm{~S}^{\prime}:=\mathrm{S}$

$? \mathrm{t}^{\prime}:=\mathrm{t}$

Now we specialise the induction hypothesis, applying it to the terms we assigned to the metavariables:

(while $\left(\lambda_{-}\right.$, true) $\left.s, t\right)=$

(while $\left(\lambda_{-}\right.$, true) $\left.\mathrm{S}, \mathrm{t}\right) \rightarrow$

false

Finally, we delete any index equation whose left-hand side is definitionally equal to its right-hand side. This leaves us with a pleasantly simple induction hypothesis:

$\mathrm{ih}_{2}$ : false

This procedure does not always succeed in eliminating the index placeholders and index equations. If we apply it to the first induction hypothesis, $i h_{1}$, it instantiates the $t^{\prime}$ index placeholder with $\mathrm{s}$, but it does not find a unique solution for $\mathrm{S}^{\prime}$. The induction hypothesis thus remains unwieldy:

$\mathrm{ih}_{1}: \forall \mathrm{S}^{\prime}$,

$(S, s)=\left(\right.$ while $\left(\lambda_{-}\right.$, true $\left.) S^{\prime}, s\right) \rightarrow$ false

This is pedagogically unfortunate, as students are unlikely to fully understand why an equation appears in $i h_{1}$. Simplifying the equation to eliminate the $s$ on both sides would help a little, but I have not encountered enough such situations in practice to justify complicating the implementation.

Besides redundant index equations, an induction hypothesis can also contain contradictory index equations, e.g. skip = while b S. The induction hypothesis can then never be applied and should be deleted. Unfortunately, my induction tactic currently does not do this, due to a limitation of Lean's built-in unification procedure, which does not allow us to distinguish between terms that are certainly unequal, such as skip and while b S, and terms that might be propositionally equal, such as $S$ and while b ? $S^{\prime}$. The Qnify procedure from Sect. 3.3 could be adapted to this use case.

\subsection{Naming of Constructor Arguments}

A surprisingly large portion of the new induction tactic is dedicated to naming. Finding intuitive names is important, particularly in an educational setting. When the names are chosen poorly, novices (and occasionally experts) may have trouble understanding how the new goals relate to the old goal. Lean's standard induction tactic uses a simple, predictable naming scheme, but the generated names are plainly too cumbersome for use in education. Consider again the fact that the transitive closure operator from Sect. 2 is transitive:

$\forall \alpha(r: \alpha \rightarrow a \rightarrow$ Type $)(a$ b $c: \alpha)$
$\quad\left(h_{1}:\right.$ tc $r$ a b) $\left(h_{2}:\right.$ tc $r$ b c $)$, tc $r$ a $c$

Performing induction on $h_{1}$, Lean's induction tactic generates a rather intimidating goal in the inductive case, shown in Fig. 5a. The goal illustrates a number of common problems:

- All new hypotheses generated by the induction tactic are prefixed with $h_{1}$. This clarifies their origin, but it also makes the goal hard to understand at a glance. Names like $h_{1 \_} x$ are simply too long compared to a plain $\mathrm{x}$.

- The first and middle elements of the transitive chain, which were called $a$ and $b$ in the lemma statement, are now called $x$ and $z$ (disregarding the $h_{1}$ prefix). One could hardly blame a novice for being confused about how the old and new hypotheses relate to each other.

- As if to make the previous problem worse, Lean's standard induction tactic does not remove the hypotheses a and $b$, even though they are now redundant and have been effectively replaced by $h_{1 \_} x$ and $h_{1 \_} z$.

Coq's standard induction tactic fares better, producing the goal in Fig. 5b. The tactic drops the $h_{1}$ prefixes and correctly removes the redundant hypotheses. Yet it, too, renames a to $x$ and $b$ to $z$.

The new induction tactic fixes this last issue, producing the goal in Fig. 5c. It recognises the connection between old and new hypotheses and names the new ones accordingly. The name $y$ is not ideal, but other than that, no name would be out of place in an informal proof. 


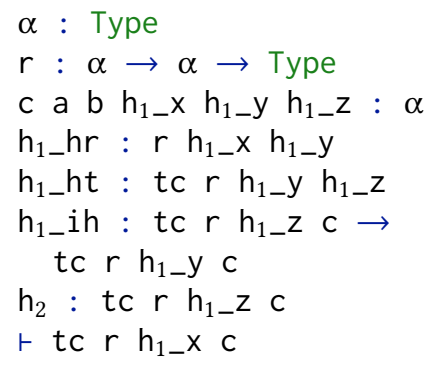

(a) Lean's standard induction tactic

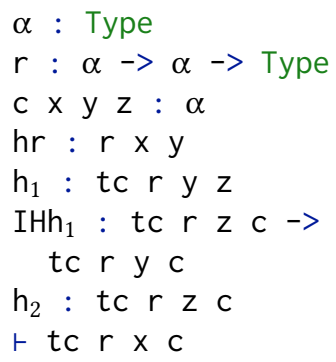

(b) Coq's standard induction tactic

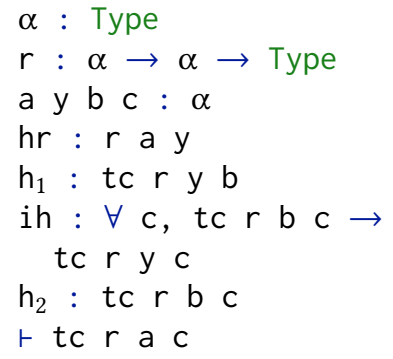

(c) The new induction tactic

Figure 5. Goals for the same proof produced by three different induction tactics

To achieve this effect, we employ the following algorithm. Suppose we are in the case of the induction corresponding to a constructor $C$. Then we need to name the following new hypotheses: one hypothesis for each of C's arguments; one induction hypothesis for each of C's recursive arguments; and any index placeholders and index equations we have introduced and not subsequently eliminated.

For the last category, a simple schema suffices. Index placeholders and index equations are usually eliminated anyway, but if they remain in the goal, we name them index_ $i$ and induction_eq_i for some $i$.

Naming the induction hypotheses is also fairly straightforward. If there is only a single induction hypothesis, we name it ih. Otherwise, we use names like ih_e, where e is the hypothesis to which this induction hypothesis applies (meaning the hypothesis corresponding to the recursive constructor argument which gives rise to ih_e). For example, if we perform induction on some expression type, we may get subexpressions $e_{1}$ and $e_{2}$ and induction hypotheses $i h \_e_{1}$ and $i h \_e_{2}$. Coq uses a similar scheme. Lean's standard induction tactic simply numbers the generated induction hypotheses, which is usually less helpful.

Finally, we consider the constructor arguments, where the naming problem becomes interesting. Suppose we want to name the hypothesis corresponding to an argument a : A of constructor $\mathrm{C}$. Then we try each rule from the following list, stopping at the first one that applies. These heuristics seem to yield intuitive names in many cases - though humans use so many different heuristics that trying to incorporate them all would be a fool's errand. When our heuristics fail, users can of course give their own names.

In larger developments, one should usually give explicit names anyway to make the proof script more robust. Still, having the induction tactic generate sensible names makes for a better user experience in the experimental phase of proof development, when the proof script is not yet polished.

Recursion. If a is a recursive argument, it is named after the major premise. So if we eliminate a natural number $n$, the number in the inductive case is also called $\mathrm{n}$; if we eliminate an expression e, its subexpressions are called e, e_1, etc. These are likely to be good names since the subexpressions are of the same type as the parent expression. In Fig. $5 c, h_{1}$ is derived from a recursive argument in this way. Coq also uses this rule.

Index association. If a is associated with an index argument, it is named after that index argument. This is the rule responsible for our improvement over Coq in the example from Fig. 5. We say that the argument $x$ of tc's step constructor is associated with the first index of tc. In the hypothesis $h_{1}$, which we are performing induction on, that first index is instantiated with $a$, so the hypothesis corresponding to $x$ is named $a$.

Capturing this situation in general requires a somewhat involved criterion. Suppose we are naming an argument a : A of a constructor $C$ whose return type is $F j_{1} \ldots j_{n}$, where $F$ is an inductive family with $n$ indices. We say that $a$ is associated with the $i$ th index if it occurs in $j_{i}$. Now suppose our major premise is e : $\mathrm{F}_{1} \ldots \mathrm{k}_{n}$. Consider those $\mathrm{k}_{i}$ such that $a$ is associated with the $i$ th index. If these $k_{i}$ are all the same hypothesis $h$, and if the type of $h$ is definitionally equal to the type of $a$, then the hypothesis corresponding to $a$ is named after $h$.

The stipulation about definitionally equal types exists to prevent confusion when a constructor argument is associated with an index of a different type. In such cases, it is usually better not to name the argument after the index, since names are often related to the types of the named entities. For instance, if an argument a : $\alpha$ is associated with an index as : list $\alpha$, we do not want the hypothesis corresponding to a to be called as. The restriction could perhaps be relaxed to allow, for instance, an argument of type list $\alpha$ to be associated with an index of type list $\beta$, but I have found no need for this in practice.

Named arguments. If $a$ is named in the definition of the constructor $C$, that name is used. In our example, the Step 
constructor has an argument called $\mathrm{hr}$, so the corresponding hypothesis is also called $\mathrm{hr}$. Coq also uses this rule.

Type-based naming. If a's type, A, is associated with a list of typical variable names, we use these. Such an association is given by an instance of the type class variable_names for A, which contains a list of names. Later, when the tactic looks for a name for $a$, it performs a type class instance search for variable_names A. If it finds an instance, it uses the first unused name from the associated list. We give such instances for some standard types (associating, for example, the names $n$ and $m$ with the type $\mathbb{N}$ ), but users can override these with their own higher-priority instances. The type class mechanism also allows us to give natural names for data structures such as lists: if a type $A$ is associated with the variable name $x$, list $A$ is by default associated with the name xs. This mechanism was developed for the new induction tactic, but can be used by other tactics as well.

Fallback. If none of the above rules apply, a receives a default name: $\mathrm{h}$ if $\mathrm{A}$ is a proposition and $\mathrm{x}$ otherwise.

The first three rules are ordered somewhat arbitrarily. I have found the given order to be the one that most often matches common naming preferences, but there are many examples where a different order would fit better. The example from Fig. 5 would arguably be improved if $h_{1}$ was called $h t$ instead, using the name from the constructor declaration rather than the recursion rule. But switching the priority of these rules would also change other names for the worse.

If the name chosen for a, say $n$, is already in use, we fall back to $n_{-} 1, n_{-} 2$, etc. This complicates the implementation because many of the induction tactic's processing steps may remove hypotheses, so we only know which names are in use after all steps have finished. To address this issue, we initially give the introduced hypotheses temporary names, then run the naming algorithm as the last step of the tactic to obtain the final names.

\section{Evaluation of Lean's Metaprogramming Framework}

I have implemented the tactic described in the previous section in the metaprogramming framework [7] of Lean 3. This provides an opportunity to evaluate how the framework fares on a moderately complex task.

\subsection{Overview of the Framework}

Like other modern metaprogramming approaches such as Mtac2 [9] or Idris's elaborator reflection [2], Lean metaprograms are written in Lean itself rather than its implementation language $\mathrm{C}++$. They are marked with the meta keyword, which signifies a stage separation: meta definitions may refer to non-meta ones, but not the other way around. Metaprograms can therefore be inconsistent (e.g. they need not terminate) without compromising the consistency of the non-meta fragment. At the same time, metaprograms have access to all the data structures and functions defined in non-meta Lean, avoiding duplicate effort.

Most metaprograms are tactics, which means they have type tactic $\alpha$ for some $\alpha$. The tactic type family is a Haskell-style monad which provides an imperative embedded domain-specific language for writing tactics. Tactics operate on a tactic state with zero or more goals. A goal has a local context, containing the current list of hypotheses, and a target type; the objective of a tactic is usually to construct an element of the target type (represented as an abstract syntax tree). To do this, tactics can make use of a large number of built-in tactics which manipulate hypotheses and the target, add and remove goals, query and add definitions, unify expressions, check whether two expressions are definitionally equal, and more.

This framework generally works well and leads to a remarkably seamless integration between regular programs and metaprograms. Still, while implementing the new induction tactic, I encountered some situations where it was less helpful or clear than it could be. The next subsections discuss these cases, which will hopefully be useful to prospective Lean metaprogrammers as well as designers of similar metaprogramming systems.

\subsection{Tracking of Hypotheses}

As mentioned, most tactics operate within a local context containing the hypotheses that are currently available. Internally, these hypotheses are represented as expressions identified by a unique name. They also have an external name which is shown to the user and is not necessarily unique in the context.

Many tactics manipulate the context in some way, e.g. by adding or removing hypotheses or changing the types of existing hypotheses. The trouble with this is that any such modification changes the unique names of any affected hypotheses. As a result, any expression involving the changed hypotheses becomes invalid: it refers to a hypothesis that, to Lean, does not exist any more.

As an example, consider the unification procedure from Sect. 3.3. It operates on a queue of index equations, unifying each in turn. Naturally, we would want to represent this queue as a list of expressions, with each expression identifying one equation hypothesis. But this does not work. Unifying the first equation may change the types of subsequent equations and thus their unique names. When we then turn to the next equation in the queue, Lean will rightfully point out that the context contains no hypothesis with that unique name. The entire tail of the queue has been potentially invalidated.

I encountered this issue multiple times - it occurs whenever one needs to track hypotheses across calls to potentially context-altering tactics, which are numerous and do not always document the fact that they may invalidate hypotheses. 
One could imagine various workarounds for this issue. For the unification procedure, I ended up identifying hypotheses not by unique name but by external name. For this to work, the external names must be unique in the context, which in this case can be ensured since the induction tactic controls these names. This workaround is less applicable when dealing with preexisting hypotheses, whose external names may not be unique. Another possible approach would be to have context-altering tactics report a mapping from old unique names to new unique names, which would allow callers to update any stored expressions. This would, however, require changes to many tactics and callers would still have to manually perform the update.

Perhaps the most convenient solution to this issue would be the introduction of yet another name for hypotheses: a stable name which would remain unchanged when a hypothesis is modified. This would most closely reflect the tactic writer's intuition that changing the type of a hypothesis does not make it a different hypothesis.

\subsection{Definitional Equality}

Any author of tactics for a dependently typed proof assistant must contend with definitional equality: different expressions that are equal up to computation. For instance, $\mathbb{N}$ and let $T:=\mathbb{N}$ in $T$ are definitionally equal types. Many tactics should treat them as interchangeable, though this depends on the tactics' use cases and user expectations. Checking for definitional equality, which involves partially normalising expressions, carries a sometimes considerable performance cost, so it would be too much to ask for a metaprogramming framework that fully abstracts over definitional equality.

Still, Lean additionally complicates the matter in two ways. First, it lacks a comprehensive programming interface for pattern-matching on expressions up to definitional equality. Tactic authors must manually normalise expressions as much as necessary, using relatively rudimentary normalisation tactics, if they want to take definitional equality into account. Novice metaprogrammers can hardly be expected to do this accurately, and experts may be tempted to cut corners and ignore the issue. This shifts the burden onto tactic users, who need to make sure that their goals have just the right shape. While implementing the new induction tactic, I added the beginnings of an up-to-definitional-equality matching framework to mathlib, but so far this covers only a few constructions.

The second way in which Lean complicates definitional equality is by introducing an additional notion of transparency. Each definition is marked with one of several transparency values, which indicate how eagerly the definition should be unfolded during normalisation. This is reasonable, and perhaps necessary: some definitions should indeed be unfolded almost always, others almost never.

However, the programming interface around transparency encourages mistakes. Most tactics which take a transparency argument make this argument optional, so if one does not supply an explicit transparency, a default value is used. This makes it easy to make subtle mistakes (and I have made a few) where transparency is not propagated or the wrong transparency is used. It does not help that different tactics have different default transparency values.

\subsection{Elaboration}

When writing a tactic, one must often construct expressions of some specific form. Lean provides essentially two ways to do this: directly, by writing out the abstract syntax tree of an expression (perhaps as a syntactically more pleasant quotation), or by elaborating a pre-expression.

Pre-expressions are an abstract syntax representation of the expressions that users write in Lean's surface syntax. They are turned into regular expressions in a process called elaboration, which fills in many details - mainly implicit and instance arguments - that users may thankfully omit.

Lean also allows us to use elaboration in tactics, which can be convenient. When writing expressions directly, we have to fill in many implicit arguments as well as universe parameters, a somewhat obscure feature of the type theory that one would usually prefer not to think about. Elaboration can do this for us, making tactics more readable and maintainable since they need only specify the main parts of an expression.

Unfortunately, Lean's programming interface again makes using elaboration more difficult than it needs to be. This is mostly due to easily avoidable limitations: many functions that construct or deconstruct expressions operate only on fully elaborated expressions, even though they could also work with pre-expressions. As a result, Lean encourages its users to elaborate early, before an expression is fully constructed. But then the elaboration algorithm lacks information about the context in which a partially constructed expression will be used, so it can infer less implicit arguments. Due to these limitations, I have usually found it more convenient to write out the fully elaborated expression after all.

\subsection{Nested and Mutual Inductive Types}

Lean takes the dictum that a proof system's kernel should be as small as possible more seriously than most dependently typed theorem provers. One ramification of this philosophy is that Lean's kernel does not support nested and mutual (collectively: generalised) inductive types. Instead, when a user writes a generalised inductive type, Lean compiles it to an equivalent non-generalised inductive type during elaboration. This approach makes the kernel smaller and thus more trustworthy. However, Lean's implementation also illustrates a major disadvantage: hiding the internal compilation process from users of the system requires much engineering effort. 
Lean does this imperfectly and as a result, generalised inductive types are a leaky abstraction. In metaprograms, the abstraction is in fact nonexistent: metaprograms only get to see the internal representation of a generalised inductive type. Thus, if a metaprogram wants to, for example, report an error about a particular generalised inductive type to the user, it has to reverse-engineer that generalised inductive type from its internal representation.

This illustrates a more general issue with tactics which act on the kernel language rather than the source language: details about the user-level program invariably get lost in translation. The induction tactic suffers from this in a small way. One of the naming rules from Sect. 3.5 checks whether a constructor argument is named in the definition of the constructor. But in the kernel language, all arguments are named, so if a user writes the constructor type $X \rightarrow Y$, our tactic sees $\forall(a: X), Y$. Thus, when the tactic encounters a nondependent argument with name a (or a_1 etc.), it assumes that this argument was not explicitly named - but that assumption can be mistaken. Perhaps the user really wrote $\forall(a: X), Y$ in the hope that our naming rule would pick up the argument name a. (The community edition of Lean 3 recently changed the elaborator so that the default name for an unnamed argument is not a but $\breve{\alpha}$, assuming that no user would choose that character as a variable name. This mitigates the issue, but $\breve{\alpha}$ now occasionally shows up in goals to confuse Lean neophytes.)

Perhaps the most effective way to prevent such loss of information would be to associate to each kernel expression the surface expression from which it was elaborated. For an inductive type, this would be the inductive declaration the user wrote; for a top-level definition, the equations given to the equation compiler. In the extreme, elaboration would become reversible, so tactics would be able to reconstruct the full program text.

Lean's treatment of generalised inductive types in metaprograms also illustrates another issue. Effectively the only primitive metaprogram that is aware of generalised inductive types is a normalisation procedure, users of which can choose whether constructors of generalised inductive types should be unfolded to their internal representation. As it happens, this is sufficient for the purposes of our induction tactic. But it shows how a feature that was supposed to be dealt with during elaboration still permeates large parts of the system: every tactic that uses normalisation must decide what to do about constructors of generalised inductive types. Given such complications, one might wonder whether it would have been preferable to put generalised inductive types in the kernel language after all.

\subsection{Open Expressions}

While the previous sections have been critical of some parts of the metaprogramming framework, this section discusses a reasonable design choice that may nevertheless be surprising to novice tactic writers: the handling of open expressions. An expression is open when it contains at least one free variable. Such expressions occur naturally when we deconstruct terms with binders. For example, consider the following type:

$\forall(n: \mathbb{N})(f:$ fin $\mathbb{N}), P n f$

We can deconstruct this type into argument types $\mathbb{N}$ and fin $\# 0$ and result type $P \# 1 \# 0$. The \# $\#$ and $\# 1$ are free variables, represented as De Bruijn indices, which refer to the variable bound by, respectively, the first and second preceding binder.

Lean lets us construct such open expressions, but it does not let us to do much with them since most built-in tactics only work on closed expressions. Open expressions cannot, for example, be type-checked or unified.

Instead, Lean's metaprogramming framework encourages users to treat expressions as locally nameless [12]. This means we effectively use hypotheses as free variables: while deconstructing an expression, we immediately replace any free variables with fresh hypotheses of the appropriate type. Our above example, so deconstructed, has argument types $\mathbb{N}$ and fin $c n$ and result type $P$ cn $c f$, where $c n: \mathbb{N}$ and $c f:$ fin $c n$ are fresh hypotheses.

This representation is considerably easier to work with, not only because Lean prefers it but also because we do not have to track the contexts of each expression as closely. Observe, for example, that in the first decomposition of our example, the $\# 0$ in the second argument and the \# 0 in the result type refer to different arguments. The locally nameless representation avoids such confusion.

There is one downside to this representation: Lean makes no particular effort to optimise the construction and deconstruction of locally nameless expressions, so these operations can be somewhat inefficient.

\section{Conclusion}

I have shown how to build a user-friendly induction tactic which is particularly suited to an educational setting. The tactic liberates its users from some of the technical, nonessential difficulties with existing induction tactics. It automatically generalises complex indices, ensuring that information contained in the indices of a hypothesis is not lost. It simplifies the resulting induction hypotheses, which would otherwise be obscured by redundant arguments. It automatically generalises induction hypotheses as much as possible so that users do not get stuck with an overly specific induction hypothesis. And it uses various heuristics to generate suitable names for all the new hypotheses it introduces. These usability improvements may spare experts some of the tedium of pre- and postprocessing their goals, and they should lift a considerable cognitive burden from novices.

I have also discussed some issues with Lean's metaprogramming framework which I encountered while implementing the new induction tactic. Some of these are easily fixable 
(but impactful) limitations of the programming interface; others point to deeper issues with aspects of the framework's design. I hope that this discussion will help make Lean's already pleasant metaprogramming even better.

\section{Acknowledgments}

Jasmin Blanchette helped determine what features a novicefriendly induction tactic should have, provided many test cases and commented in great detail on drafts of this paper. Anne Baanen, Floris van Doorn, Gabriel Ebner, Rob Lewis and the anonymous reviewers gave detailed and insightful feedback on drafts of this paper and on the underlying code. The Lean Zulip community, particularly Mario Carneiro and Gabriel Ebner, patiently answered my many questions about Lean metaprogramming. Many thanks!

This project was funded by the NWO under the Vidi programme (project No. 016.Vidi.189.037, Lean Forward).

\section{References}

[1] Mario Carneiro. 2019. The Type Theory of Lean. Master's thesis. Carnegie Mellon University, https://github.com/digama0/lean-typetheory/.

[2] David Christiansen and Edwin Brady. 2016. Elaborator Reflection: Extending Idris in Idris. In Proceedings of the 21st ACM SIGPLAN International Conference on Functional Programming (Nara, Japan) (ICFP 2016). ACM, New York, 284-297. https://doi.org/10.1145/2951913.2951932

[3] Jesper Cockx, Dominique Devriese, and Frank Piessens. 2014. Pattern Matching without K. In Proceedings of the 19th ACM SIGPLAN International Conference on Functional Programming (Gothenburg, Sweden) (ICFP '14). ACM, New York, 257-268. https://doi.org/10.1145/2628136. 2628139

[4] Jesper Cockz and Dominique Devriese. 2018. Proof-Relevant Unification: Dependent Pattern Matching with Only the Axioms of Your
Type Theory. Fournal of Functional Programming 28 (2018), e12. https://doi.org/10.1017/S095679681800014X

[5] The mathlib Community. 2020. The Lean Mathematical Library. In Proceedings of the 9th ACM SIGPLAN International Conference on Certified Programs and Proofs (New Orleans) (CPP 2020). ACM, New York, 367-381. https://doi.org/10.1145/3372885.3373824

[6] Peter Dybjer. 1994. Inductive Families. Formal Aspects of Computing 6, 4 (1994), 440-465. https://doi.org/10.1007/BF01211308

[7] Gabriel Ebner, Sebastian Ullrich, Jared Roesch, Jeremy Avigad, and Leonardo de Moura. 2017. A Metaprogramming Framework for Formal Verification. Proc. ACM Program. Lang. 1, ICFP, Article 34 (Aug. 2017). https://doi.org/10.1145/3110278

[8] Martin Hofmann and Thomas Streicher. 1994. The Groupoid Model Refutes Uniqueness of Identity Proofs. In Proceedings of the Ninth Annual IEEE Symposium on Logic in Computer Science. IEEE, 208-212. https://doi.org/10.1109/LICS.1994.316071

[9] Jan-Oliver Kaiser, Beta Ziliani, Robbert Krebbers, Yann Régis-Gianas, and Derek Dreyer. 2018. Mtac2: Typed Tactics for Backward Reasoning in Coq. Proc. ACM Program. Lang. 2, ICFP, Article 78 (July 2018). https://doi.org/10.1145/3236773

[10] Conor McBride. 1996. Inverting Inductively Defined Relations in LEGO. In International Workshop on Types for Proofs and Programs. Springer, Berlin/Heidelberg, 236-253. https://doi.org/10.1007/BFb0097795

[11] Conor McBride. 2002. Elimination with a Motive. In Types for Proofs and Programs, Paul Callaghan, Zhaohui Luo, James McKinna, and Robert Pollack (Eds.). Springer, Berlin/Heidelberg, 197-216. https: //doi.org/10.1007/3-540-45842-5_13

[12] Conor McBride and James McKinna. 2004. Functional Pearl: I Am Not a Number-I Am a Free Variable. In Proceedings of the 2004 ACM SIGPLAN Workshop on Haskell (Snowbird, USA) (Haskell '04). ACM, New York, 1-9. https://doi.org/10.1145/1017472.1017477

[13] Benjamin C. Pierce, Arthur Azevedo de Amorim, Chris Casinghino, Marco Gaboardi, Michael Greenberg, Cătălin Hriţcu, Vilhelm Sjöberg, and Brent Yorgey. 2020. Software Foundations Vol. 1: Logical Foundations. https://softwarefoundations.cis.upenn.edu.

[14] The Univalent Foundations Program. 2013. Homotopy Type Theory: Univalent Foundations of Mathematics. https://homotopytypetheory. org/book. 